\title{
The Role of Calcium Phosphates and Electrospun 3D Scaffolds in Bone Tissue Engineering Scaffolds
}

\author{
V. Yogeshwar Chakrapani, T. S. Sampath Kumar
}

\begin{abstract}
Bone is a naturally occurring nano-composite structure bestowed with an innate regenerative potential. When this regenerative potential is not able to cope up with the bone loss, external assistance in the form of scaffolds, cells and signals are needed. This forms the basis of bone tissue engineering (BTE). $\mathrm{CaP}$ ceramics like hydroxyapatite $(\mathrm{HA})$, calcium deficient hydroxyapatite $(\mathrm{CDHA})$ and $\beta$-tricalcium phosphate $(\beta-T C P)$ are an excellent choice of material for hard tissue reconstruction. However, they are brittle in nature and solid ceramic constructs are not conducive for vascularisation, thus limiting their application as scaffolds for BTE. Thus composite scaffolds of appropriate polymer/ceramic combination would greatly benefit BTE. Electrospinning is an extremely versatile methodology that is predominantly used for the fabrication of nanofibrous structures that closely mimic the ECM. Nevertheless, electrospinning of $3 D$ structures is still a challenge. Various innovations in the electrospinning process are being tried out in order to produce true 3 dimensional structures that can act as scaffolds for BTE. The current paper reviews such technologies and also suggests the way forward for research in this area.
\end{abstract}

Keywords: $3 D$ structures, Bone tissue engineering, Electrospinning

\section{INTRODUCTION}

Mammalian tissues are chiefly classified into: epithelial, connective, muscle and nervous. A well defined and an elaborate arrangement between these tissues form the diverse organs of the body and the flawless connections among them. An optimal functioning of these arrangements is essential for leading a healthy life. Majority of the tissues consist of more than one cell type and the extracellular matrix (ECM) components that are secreted by these cells. The ECM plays a major role in the regulation of various cellular functions such as, adhesion, proliferation, migration, differentiation and matrix deposition [1].

\section{A. Bone biology and structure}

Bone is known to be a specialised connective tissue that is responsible for many important functions such as providing the body a framework, load bearing, locomotion and protecting the viscera. Furthermore it also has a crucial reservoir function, as the storehouse of the essential minerals for the human body.

Revised Manuscript Received on December 15, 2019.

* Correspondence Author

Dr. V. Yogeshwar Chakrapani, Associate Professor Department of Instrumentation And Control Engineering Kalasalingam Academy of Research And Education, Krishnankoil, India.

Dr. T. S. Sampath Kumar, Professor Department Of Metallurgical And Materials Engineering, IIT Madras.

It houses the bone marrow, a vital stem cell niche in the human body, which produces and stores most of the red blood cells, white blood cells, fat and bone tissue itself. It is also the most prolific tissue in the body, constantly remodeling itself depending on the various mechanical and biological stimuli.

Bone is a naturally occurring nano-composite structure bestowed with an innate regenerative potential, without scar formation [2], which becomes active after fracture or other bone loss related injuries.

The predominant cell types responsible for the structural and functional integrity of the bone tissue are osteoblasts, bone lining cells, osteocytes and osteoclasts. These are embedded in an intricate matrix that consists of inorganic and organic components. Ninety percent of the organic matrix is made up of collagenous proteins (primarily type I collagen). The rest is made up of non-collagenous proteins including osteopontin, osteocalcin, osteonectin, fibronectin and bone sialoprotein II, bone morphogenetic proteins (BMPs), and several growth factors [3]. The inorganic component of bone known as biological apatite, consists largely of calcium and phosphate ions; however, significant amounts of sodium, potassium, bicarbonate, carbonate, citrate, magnesium, fluorite, barium, zinc and strontium are also present [4]. Calcium and phosphate ions nucleate to form the hydroxyapatite (HA) crystals. The unique organic scaffold and the organized deposition of $\mathrm{HA}$ in it results in the distinctive stiffness of the bone tissue [3].

\section{B. Bone loss (Skeletal pathology)}

Bone loss is a major world-wide problem with over 7.9 million fractures, out of which 5-10\% result in delayed healing or non-union, estimated annually in the USA alone [5]. Trauma leading to bone loss cost a staggering 21 billion dollars for treatment in the USA. Though recent, updated studies reporting the numerical statistics of bone related injuries and their treatment pertaining to Indian population are scarce, bone injuries and infections are a serious health issue in the Indian subcontinent too. After blood, bone is the most transplanted tissue [6]. Bone loss can be attributed to a variety of reasons such as age related issues, trauma, lifestyle, physiological \& congenital disorders and other miscellaneous factors. All these factors disrupt the bone homeostasis, by affecting the bone remodeling, bone resorption or bone deposition, in one way or the other. These factors have been classified and shown diagrammatically in Fig. 1. 
Congenital factors that result in deformed bones, abnormal or stunted bone growth are a leading cause of skeletal pathologies. Examples include brittle bone disease, stone man syndrome and hypopitutarism leading to dwarfism in children. Genetic mutations also lead to osteopetrosis, characterized by retarded bone resorption resulting in increased bone mass and density due to defective functioning of osteoclasts [7]. Fractures are the most common and highly prevalent cause for bone injuries. They can occur due to trauma and accidents or due to the aging process. Aging results in natural loss of bone density (osteoporosis) and an increased incidence of falling, both of which may lead to fractures. Fractures can either be open, rupture in skin and muscles, or closed, no rupturing of skin. For open fractures, chances of infections occurring and spreading are higher. Based on the way a bone splits, fractures are classified into various types as shown in Fig. 1.

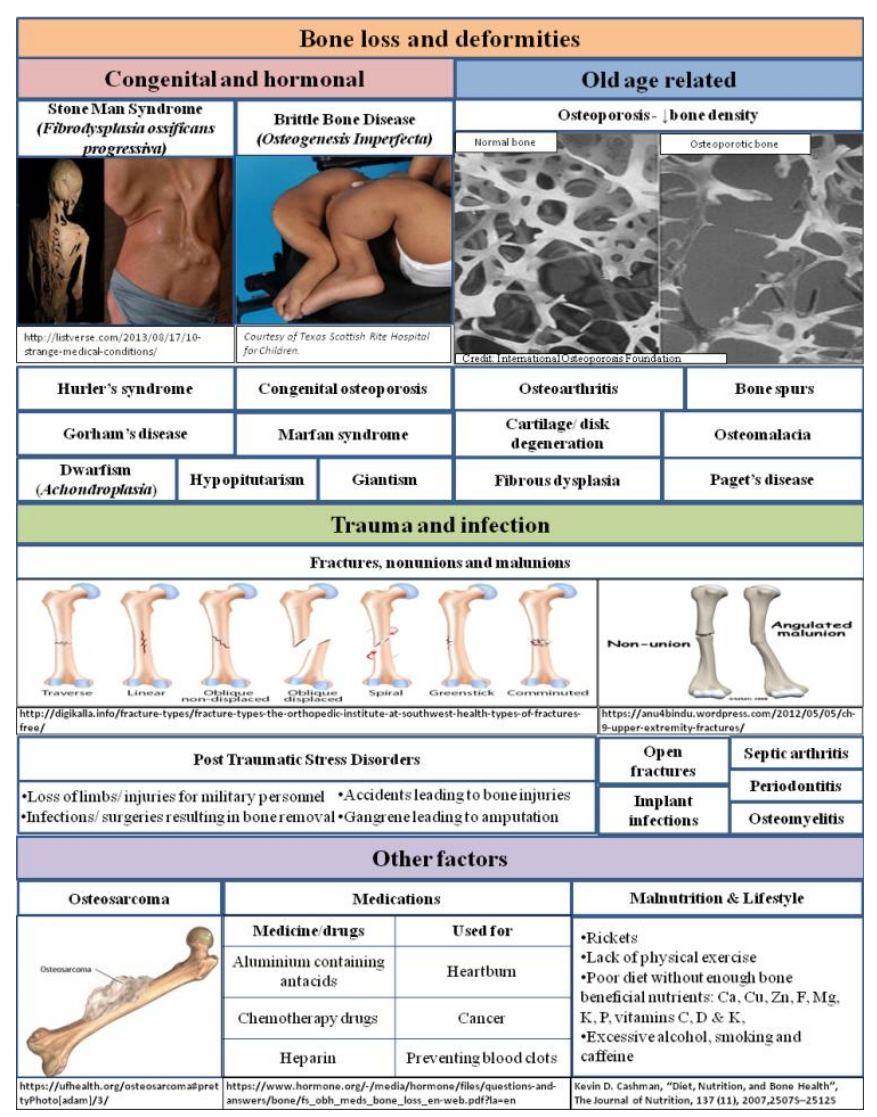

Fig. 1.Causes for bone loss and deformities in the human body

When the balanced dynamic functioning of the bone tissue is upset, bone diseases start to manifest. Osteoporosis is one such disease where due to abnormal osteoclast activity, bone resorption exceeds bone formation. This leads to drastic reduction in bone density that subsequently results in bone fractures [8]. Similarly in periodontitis, a dental disease caused by bacterial proliferation, there is increased bone resorption, resulting in loss of teeth insertions and other complications [3]. Gangrene leading to amputate ions and procedures requiring the removal of necrotic/ cancerous (osteosarcoma) bony tissues also lead to bone loss. Medications taken for various conditions such as cancer, blood clot prevention, etc. are also known to adversely affect bone metabolism and subsequent bone loss. Other factors like calcium deficient diet, absence of activities that load bones so as to enhance the remodeling phase and excessive smoking and drinking are also known to affect bone density.

\section{BONE TISSUE ENGINEERING}

When there is disruption in the bone tissue architecture and function, as a result of trauma, disease, or wear and tear, the first line of defense is the body's innate healing mechanism. Briefly, a blood clot forms over the wound, and the necessary growth factors, stem cells, etc. are transported via blood, to the precise site of injury and the new tissue formation begins. When such a natural process becomes inadequate or compromised due to various causes, external intervention becomes necessary to restore and maintain the structural and biological functions of the tissue, through tissue reconstruction or tissue substitutes [9]. Although autografts and allografts are the golden standards for bone substitutes, they are plagued by drawbacks such as donor site morbidity, limited availability, risk of infection and/or mismatch of mechanical properties. This has prompted interest in developing external regenerative resources including scaffolds, cells and growth factors (GF) that are employed, either alone or in combination. They are commonly depicted in literature in the form of the tissue engineering (TE) triad (Fig. 2).

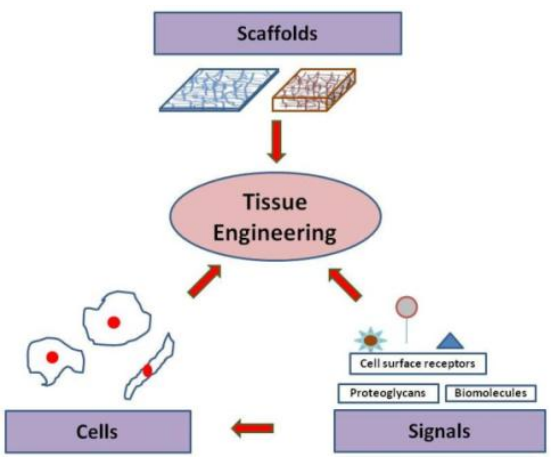

Fig. 2. The tissue engineering triad

The need of the hour are artificial bone grafts that are bioactive, porous in nature, capable of withstanding the cyclic loading action along with the biological and structural cues necessary for osteogenesis. The biological cues include bone like cells, growth factors and other biomolecules that instigate and sustain bone growth. It is now acknowledged that the cell-scaffold interactions form a vital part of any tissue engineering strategy. The science and technology involved in producing and testing such scaffolds/constructs can be collectively termed as bone tissue engineering (BTE).

BTE includes the technologies of creating bioactive fillers and replacements for injured bones, scaffolds for augmenting bone re-growth, targeted drug delivery systems for healing bone related injuries etc. The common strategy of TE involves the usage of undifferentiated or specialised cells seeded inside a scaffold that imitates the geometry of the concerned tissue (bone) and gives the environmental cues that support the growth of new tissues [1].

Published By: 
Additionally, trying to mimic the biological milieu, namely the various growth factors and cell surface receptors involved in new bone formation, is also an approach adopted in BTE

\section{CALCIUM PHOSPHATES FOR BTE}

Calcium phosphate $(\mathrm{CaP})$ compounds are a group of inorganic materials that comprise of calcium $\left(\mathrm{Ca}^{2+}\right)$ and phosphate $\left(\mathrm{PO}_{4}{ }^{3-}\right)$ ions along with oxygen $\left(\mathrm{O}^{2-}\right)$ and hydroxyl $\left(\mathrm{OH}^{-}\right)$ions. Calcium phosphate $(\mathrm{CaP})$ ceramics, due to their compositional similarity with the inorganic components of hard tissues [5], have been a preferred material for the reconstruction of hard tissues [10], bone substitutes/fillers, TE scaffolds, bone and dental cements and coatings on bone implants. Moreover the possibility of tailoring their degradation by varying the $\mathrm{Ca} / \mathrm{P}$ ratio widens their scope of applications. The role of $\mathrm{CaPs}$ in enhancing bioactivity and influencing cellular proliferation and differentiation, when used in the form of ceramic scaffolds or as an inorganic component in composite scaffolds are well documented [11]. They are also known to be capable of inducing osteogenic cell differentiation and osteoconductive [12]. For dental cements applications a variety of combinations of CaPs, like dicalcium phosphates (brushite, monetite) and tetracalcium phosphates, are studied extensively. Additionally, the capability of $\mathrm{CaP}$ particles to adsorb drug and other bio-molecules, has made them a viable candidate for acting as carriers for drug, protein and gene delivery applications [13]. Further, reconstruction of bone defects prior to implant surgery also benefit from the use of $\mathrm{CaP}$ particles such as $\beta$-TCP, due to their bone remodeling capacity, which is related to their enhanced degradation compared to other $\mathrm{CaP}$ ceramics [14]. CaPs like HA, are also used as inorganic fillers to form electrospun composites.

The various $\mathrm{CaPs}$ often reported in literature for orthopaedic and dental applications, are listed in Table 1. Among these CaPs, hydroxyapatite (HA), calcium deficient hydroxyapatite (CDHA) and $\beta$-tricalcium phosphate $(\beta$-TCP) are widely studied as bone substitutes and fillers in composites for BTE scaffolds. Among other variations, $\mathrm{CaP}$ compounds vary in terms of their calcium to phosphate ratios $(\mathrm{Ca} / \mathrm{P})$. While $\mathrm{HA}$ with a $\mathrm{Ca} / \mathrm{P}$ of 1.67 is the most stable apatite, $\mathrm{CDHA}$ is bioresorbable and its resorption rate can be tailored by altering the $\mathrm{Ca} / \mathrm{P} . \beta$-TCP is known as a metastable member of the CaP family [15]. It is a high temperature phase of $\mathrm{CaPs}$ that cannot be precipitated from aqueous solutions. It is generally prepared by the thermal decomposition of other $\mathrm{CaPs}$, at temperatures above $800{ }^{\circ} \mathrm{C}$ [15]. Technically this involves a transformation of CDHA to $\beta$-TCP. This transformation is dependent on the extent of Ca deficiency present in the synthesized CDHA or in other words, the value of ' $x$ '(in the formula for CDHA in Table 1) or amount of $\left(\mathrm{HPO}_{4}\right)^{2-}$ introduced into the lattice structure of CDHA [16].

Artificial bone grafts include bioactive ceramic materials made from HA, $\beta$-TCP, etc. These ceramics are preferred for bone filler applications due to desirable properties such as bioactivity, osteoconduction and osteoinduction. However, they are brittle in nature and solid ceramic constructs are not conducive for vascularisation, thus limiting their application

as scaffolds for BTE. Pure ceramics cannot replicate a bone's structure and functionality entirely. Polymeric materials, like polycaprolactone (PCL), are able to incorporate the elasticity, mouldablity and porosity necessary for bone tissue engineering scaffolds, but lack inherent bioactivity and osteoinductivity. Hence polymer/ ceramic composite materials are preferred in order to fabricate scaffolds that closely mimic the natural bone ECM.

\section{SCAFFOLDS FOR BTE}

The primary goal of TE lies in creating micro-environments that effectively sustain cell growth, without adversely affecting the functionality of the wounded tissue. This is largely influenced by the scaffolds which act as the synthetic ECM. Ideally these scaffolds should be capable of hosting a milieu that is conducive for cellular attachment, growth and ultimate development into the intended tissue [17]. When dealing with BTE, the structural and functional aspects of the bone needs to be taken into account during scaffold fabrication. Recent studies are increasingly suggesting that three dimensional (3D) scaffold structures have a critical role in inducing cellular organization and ultimately mimicing anisotropic tissues, such as cardiac muscle or bone $[18,19]$.

Table 1 Calcium phosphates used for biomedical applications

\begin{tabular}{|c|c|c|c|}
\hline Sno. & CaP compornd & Chemical formina & $\begin{array}{l}\text { Ca/P } \\
\text { molar } \\
\text { ratio }\end{array}$ \\
\hline 1. & $\begin{array}{lr}\text { Dicalciimm phoshate } \\
\text { dilyydrate } \\
\text { mineral brushitt }\end{array}$ & $\mathrm{Ca}\left(\mathrm{H}_{2} \mathrm{PO}_{4}\right)_{2}-\mathrm{H}_{2} \mathrm{O}$ & 1.0 \\
\hline 2. & $\begin{array}{|lr|}\text { Dicakiim } & \text { phosphate } \\
\text { antyduns } & \text { (DCPA) } \\
\text { mineral monetitte }\end{array}$ & $\mathrm{Ca}\left(\mathrm{H}_{2} \mathrm{PO}_{4}\right)_{2}$ & 1.0 \\
\hline 3. & $\begin{array}{l}\text { Octacalcimm phosphate } \\
\text { (OCP) }\end{array}$ & $\mathrm{Ca}_{8}\left(\mathrm{HPO}_{4}\right)_{2}\left(\mathrm{PO}_{4}\right)_{4}-5 \mathrm{H}_{2} \mathrm{O}$ & 1.33 \\
\hline 4. & $\begin{array}{l}\text { a-Thicalcim phosphate } \\
\text { (a-TCP) }\end{array}$ & $a-\mathrm{Ca}_{3}\left(\mathrm{PO}_{4}\right)_{2}$ & 1.5 \\
\hline 5. & $\begin{array}{l}\text {-Thicalcinm phosphate } \\
\text { (B-TCP) }\end{array}$ & $\beta-\mathrm{Ca}_{3}\left(\mathrm{PO}_{4}\right)_{2}$ & 1.5 \\
\hline 6. & $\begin{array}{l}\text { Amomphous calcinm } \\
\text { phosphate(ACP) }\end{array}$ & $\begin{array}{l}\mathrm{C}_{2} \mathrm{H}_{3}\left(\mathrm{PO}_{4}\right)_{2}-\mathrm{nH}_{2} \mathrm{O}, \mathrm{n}=3-45 \\
15-20 \% \mathrm{H}_{2} \mathrm{O}\end{array}$ & $1.0-2.2$ \\
\hline 7. & $\begin{array}{l}\text { Calcinm-deficient } \\
\text { hydroxyapatite (CDHA) }\end{array}$ & $\begin{array}{l}\mathrm{Ca}_{10-x}\left(\mathrm{HPO}_{4}\right)_{x}\left(\mathrm{PO}_{4}\right)_{6-x}(\mathrm{OH})_{2=} \\
(0<x<1)\end{array}$ & $1.5-1.67$ \\
\hline 8. & Hydroxyapatite (HA) & $\mathrm{Ca}_{10}\left(\mathrm{PO}_{4}\right)_{0}(\mathrm{OH})_{2}$ & 1.67 \\
\hline 9. & $\begin{array}{lr}\text { Tetracalcium } & \text { phosphate } \\
\text { (TICP), } & \text { mineral } \\
\text { hilgenstinckite } & \end{array}$ & $\mathrm{C}_{4}\left(\mathrm{PO}_{4}\right)_{2} \mathrm{O}$ & 2.0 \\
\hline
\end{tabular}

Scaffold architecture at the micro level is identified as having a critical role in guiding $3 \mathrm{D}$ cellular organization and the subsequent recuperation of the tissue and its functions [20]. Hence apart from analyzing the cellular interactions with the scaffolds, it becomes essential to study and understand the structural development of the scaffold architecture in order to come up with innovations in engineering novel scaffolds [21]. 
It comes as no surprise to realize that in comparison to the studies focused on understanding and replicating the chemical milieu in which cells reside, there are fewer efforts in the direction of unraveling the relation between architectural cues and cell growth [21]. Hence conventional techniques of scaffold fabrication are either being upgraded based on the expanding knowledge base of cell scaffold interactions or replaced by more novel techniques. The various techniques for fabricating 3D scaffolds are shown in Fig. 3.

The methods shown in a darker brown background are the conventional methods. The limitations often associated with such conventional scaffold fabrication techniques [22] are listed below:

1. Poor or lack of control over the pore geometries, distribution and interconnectivity.

2. Thickness of scaffolds is very less making cross section examination difficult.

3. The removal of residual organic solvents that can become toxic to the cells being cultured.

4. Most of the end products resemble foam structures, which lack sufficient passageway for the nutrients to the interiors, thereby obstructing the movement of cells into the structure

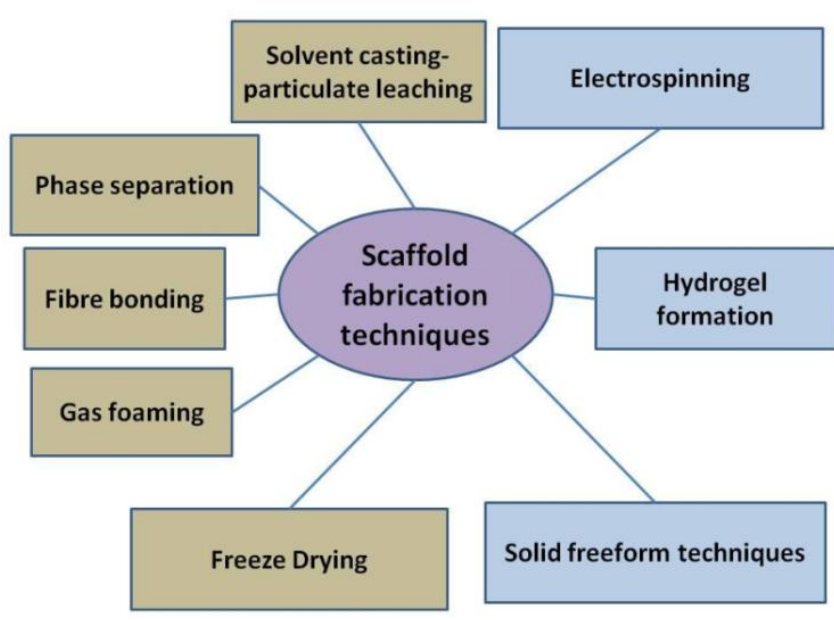

Fig. 3.3D scaffold fabricating techniques

Solid freeform fabrication (SFF) techniques collectively refer to manufacturing strategies that utilize the layer by layer building techniques to produce 3D structures. They are commonly also referred to as rapid prototyping techniques. Even though the individual process may vary, the basic principle involved is similar: producing a $3 \mathrm{D}$ corporeal structure based on a simulated model through the data obtained either from a CT (computed tomography) scan or an MRI (magnetic resonance imaging) or a CAD (computer aided design) [22]. The ability to accommodate an artificial vascular system while building the 3D structure along with the accurate control over parameters such as pore size, porosity and pore distribution, which increases the success rate of synthetic scaffolds, are a couple of attractive features of SFF techniques. In the past couple of decades more than 20 SFF technologies have been developed and commercialized [22]. However SFF techniques are also burdened by shortcomings, such as costly/ complex equipment and the limited number of materials that can be processed by them.
Hydrogels are insoluble cross-linked hydrophilic polymer networks, preferred for bone regenerative applications, that swell by absorbing huge amounts of water. A large number of natural and synthetic polymers are commonly used as raw materials for forming hydrogels. The extensive presence of water in them, improves their biocompatibility over bulk polymers and the porous environment provide a passage for cellular migration and proliferation. Their structure replicate the hydrated structural feature of the naturally occurring ECM [23]. Injectable hydrogels are ideal candidates for some TE applications because of their ability to fill unevenly shaped tissue defects. Hydrogels are also capable of handling the stresses associated with mechanotransduction [24] and are easier to handle and incorporate cells and bioactive agents [25]. Yet, one of the predominant drawbacks of a hydrogel matrix is that its matrix does not have a cellular-scale architecture that is essential for encouraging cellular adhesion and subsequent cellular infiltration necessary for the tissue repair [26]. Finally the mechanical vulnerability of a standard hydrogel limits its applications as an alternative for load bearing functions.

Electrospinning has emerged as a multipurpose fabrication technique, in the past 3 decades, for producing nanofibrous structures which have potential applications in the TE domain. Among the various material types, those of fibrous nature are more desirable for TE applications. This can be attributed to their high surface area to volume ratio, high porosity and most importantly, their architectural similarity to the natural ECM [27]. 3D nanofibrous environments help in creating scenarios favorable for the in vivo like cellular phenotypes and overall tissue morphogenesis [28]. A synthetic ECM should not only be similar to natural ECM in terms of the morphological features but also possess the biomechanical and biochemical properties that are essential for tissue development and overall homeostasis [29]. The submicron/ nano dimensions and the ultrafine nature of the fibres, makes them an ideal substrate for cellular adhesion and proliferation. Specific functionalization of the surface of the fibres so as to make it favorable for a particular cell type also becomes a viable option. Loading of biomolecules or drugs onto the fibres to increase its range of applications in TE also becomes a practical option.

Electrospraying, a technique closely related to electrospinning, is also emerging as one of the preferred methods for producing particles in the micro/nano ranges, with uniform size distribution. It is being studied for various therapeutic and regenerative applications in tissue engineering. Polymeric materials are widely processed using electrospraying, while ceramic materials are yet to be explored in this regime completely.

The following section will give a brief overview of the history, fundamentals and evolution of electrospinning and electrospraying techniques. The innovations in electrospinning aimed at producing 3D structures have also been have also been reviewed from literature.

\section{ELECTROSPINNING AND ELECTROSPRAYING}

The studies on the effect of high electric currents on pointed conductors and those on liquids/ polymeric solutions formed the basis for what is currently known as electrospinning/ 
electrospraying. The earliest mention of an electric effect on a liquid menisci, in literature is by William Gilbert in 1600. A report in 1882 by Rayleigh (Rayleigh 1882) describing the effect of electrical charges on spherical liquid droplet, 5 patents by Coley, Morton and Zeleny and the 4 scientific publications by Zeleny during the early $20^{\text {th }}$ century laid the basis for the concept of electrohydrodynamic atomization (EHDA). EHDA was initially confined to studies on liquids and took more than half a century to be applied to ceramic suspensions, polymeric solutions and melts and recently to ceramic precursors. This period resulted in quite a few patents, publications and reviews after which the terms electrostatic spinning and finally electrospinning became a standard term used in literature. Electrospraying was studied more for aerosols production and focus was on explaining the mode of jetting resulting when a high voltage was applied. Last 2 decades have seen immense growth in electrospraying for micro and nanoparticle production for coating, drug delivery and powder forming technologies. A flow chart of a few significant patents/ reports/ publications on the phenomenon of "electrified liquid droplets" leading to the present day mainstream techniques of electrospinning/ electrospraying is shown in a chronological order in Fig. 4.

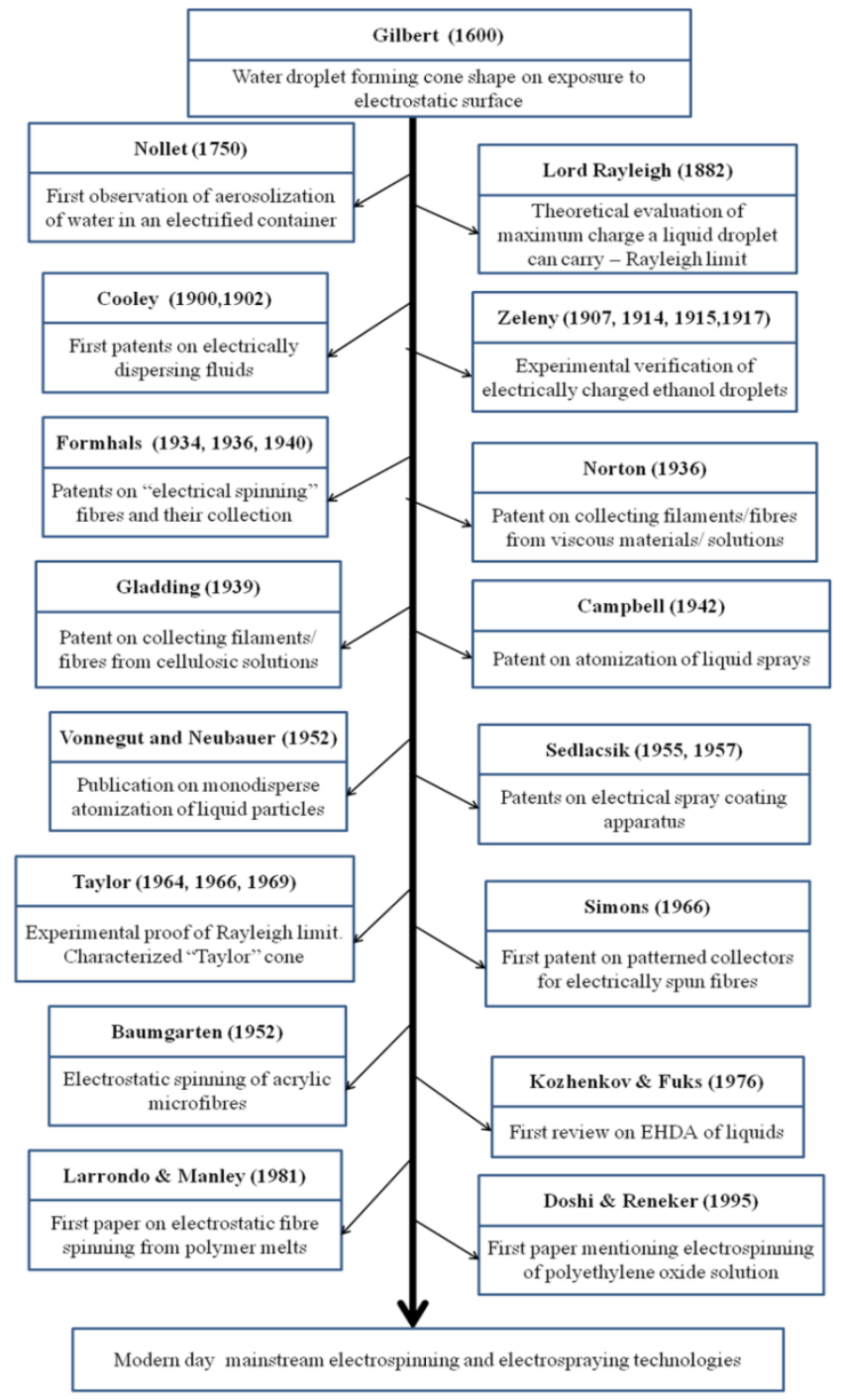

Fig. 4.Flow chart showing the evolution of electrospinning/electrospraying technologies
Electrospinning is an electrohydrodynamic technique that specialises in fabricating of thin (micro/ nano) continuous fibres, by subjecting polymer solutions or melts to high voltages. When this phenomena, is utilised for the production of micro/nano particles it shifts into the electrospraying domain. In fact, both the processes are strongly influenced by the balance between the viscous forces and the surface tension of the polymeric solution [30]. By varying the process parameters and the solution properties, it is possible to produce fibres, beaded fibres and particles of varying morphologies such as spheres, porous particles, cups, etc. For example, a low polymer concentration (meaning a low viscosity), leads to the formation of beads, while a gradual increase in the concentration often produces beads-on-fibres structure and ultimately the formation of fibres at the optimized concentration [31]. The emergence of electrospraying, as a commonly favored particle synthesis technique is because of its superior control over size distribution which results in monodisperse particles [32]. Such particles are known to be beneficial in producing a reproducible and controlled loading and release profiles when used for drug delivery applications [33].

The conventional electrospinning/ electrospraying, as shown in Fig. 5, is comprised of the following components:

- A solution reservoir

- A spinneret

- A high voltage setup and

- A collector for collecting the electrospun fibres/ electrosprayed particles.

The basic process parameters that influence the final outcome of electrospinning are: solute concentration, spinneret tip to collector distance, pumping/flow rate of the solution and the applied voltage.

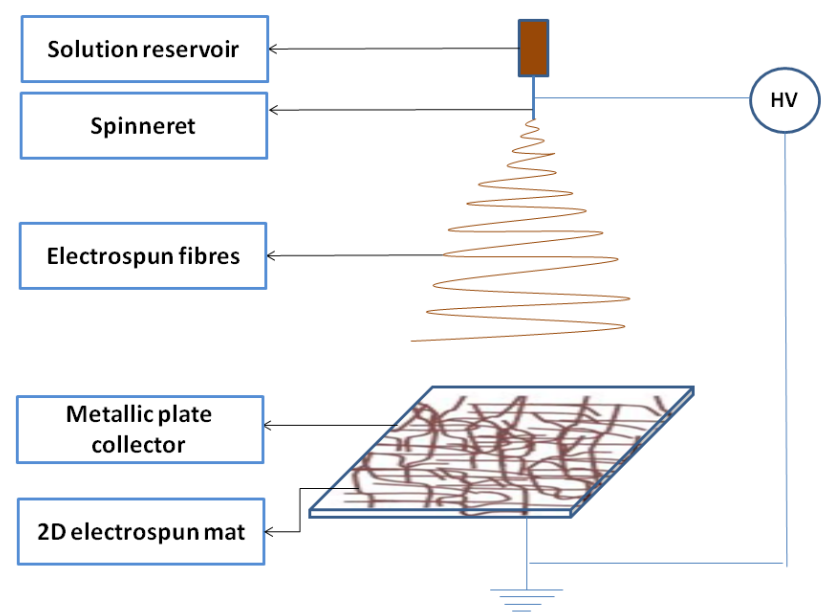

Fig. 5.Conventional electrospinning setup

The reasons behind the widespread usage of electrospinning in fabricating tissue engineering scaffolds are summarised as below:

a. The relatively simple and cost effective process of setting up an electrospinning system.

b. The fundamental unit of a conventional electrospun structure, the 'nanofibre' has an exeptionally high surface area to volume ratio. This makes the electrospun structure resemble the fibrous networks of the natural ECM [34] thus making it favorable for cell growth and proliferation. 
c. The ability to process a wide range of materials including synthetic and natural polymers and polymerless sol-gel systems [35]

d. The prospect processing polymer blends, (e.g. synthetic with natural polymers). This in turn aid in overcoming the inherent drawbacks of individual polymers and combining the desirable features of both the polymers.

e. The flexibility available in producing different structures by either varying the collecting methods or through post processing techniques. The various types of collectors reported include rotating mandrels, metallic plates, collectors with air gaps, patterned surfaces, liquid bath collectors etc.

f. The ever present scope for expanding the versatility in producing novel electrospun structures through system modifications and innovative control of process parameters.

g. The possibility of functionalizing the fibres by the addition of ceramic nano fillers, drugs growth factors, etc.

h. The opportunity to expand large scale cell culture protocols by introducing the usage of nanofibres in cell reactors/ roller cultures and the usage of electrospun structures as scaffolds for ex vivo culturing of cells to be used in TE and other cell based therapies.

There has been a tremendous growth in the applications of electrospinning in various sectors such as;

- Medicine-medical textiles, tissue engineering scaffolds

- Filtration - micro/nano filters for air purification

- Energy - battery related applications, etc.

However, majority of the studies focus on utilising the conventional electrospinning setup with minor variations and are typically able to form only two dimensional (2D) non-woven mats. Achieving fibre alignment, porous fibres and true three dimensional (3D) structures which are better substitutes for the natural ECM, remains a concern.

\section{ElECTROSPUN 3D STRUCTURES AS TISSUE ENGINEERING SCAFFOLDS}

Despite its nanofibrous morphology, a conventional 2D electrospun mat is unable to mimic the natural ECM in terms of thickness, hierarchical fibre organisation, tissue specific profile and contour and pore dimensions that favour cellular infiltration [34]. A report highlights that 3D structures mimic the native ECM more closely by creating the appropriate microenvironment (in terms of structure and porosity) and the essential mechanical cues for aiding the growth and proliferation of cells used in TE [35]. The two prime limitations of electrospinning are a) the whipping instabilities leading to the chaotic/ random fibre deposition and b) the difficulty in fabricating three dimensional self supporting structures. There is a plethora of studies, in recent times, focussed on innovative electrospinning techniques and setups that are claimed to be capable of producing novel structures which can be used as TE scaffolds [35, 36]. For achieving this, either post processing of 2D non-woven mats or enhancement in the process parameters and/or the collector systems have been attempted.

Post processing of conventional electrospun mats has been carried out to generate more porous and thicker structures. Straighforward methods include forming tubular structures by rolling/folding the electrospun mats [37] and preparing high porosity mats through the mechanical expansion of

conventional electrospun mats, with the help of metallic combs. During mechanical expansion, though the overall structure becomes more porous and fluffier, the process of forcefully disrupting a stable architectural arrangement can be degrading to the structure and make the resultant structure fragile and unstable.

Electrospun 3D structures were also achieved through modification of the electrospinning process methodologies and by manipulating the electrostatic properties. Basic methods to increase an electrospun mat's thickness include increasing the fibre deposition time and multi-layering. Multi-layering has been achieved through co-electrospinning, sequential electrospinning and also through the use of supplementary electrical fields for focussing the electrospun fibres into a confined collecting space. Metallic rings have been used as auxiliary electrodes during such processes [38]. This resulted in focussing the electrospun fibres into a smaller region and subsequently increasing the thickness of the obtained electrospun mat. Another favourable feature obtained was the hierarchical porous structure. The presence of nanofibres between the microfibres favoured the spreading of mesenchymal stem cells (MSC) [39]. Similarly, nanofibres electrospun along with microfibres showed considerably higher adhesion and viability rates of MSC, than when nanofibres alone were tested [40]. The addition of salt during electrospinning and its subsequent removal (salt leaching) afterwards, has resulted in a porous 3D structure [41]. Biological assessment showed significant chondrocyte adhesion and proliferation, while maintaining the characteristic roundness of chondrocytes. Fluffy web like structures made of polyacrylonitrile/ multiwall carbon nanotubes composite fibres, with low packing density, have been reported to be formed with the help of metallic discs as supporting electrodes during electrospinning [42]. Another avenue explored involves controlling the repulsion between the electrospun fibre and the collector surface. This has been achieved by the inclusion of sodium dodecyl sulphate (SDS) which reduced the surface resistivity of polymer and resulted in a loosely packed fibrous structure. On similar lines, conical spongiform structures have been self assembled by manipulating the electrostatic forces between the electrospun polystyrene fibres and electrospinning jet [43]. Electrospun PCL fibres have also been self assembled into honeycomb structures [44]. This was achieved by focussing the fibre deposition by using an insulating mask above the metallic collector.

Another method of producing electrospun 3D structures involves the use of custom made collectors aimed at innovative ways of collecting the electrospun fibres. These include both solid and liquid assemblies. Static and dynamic collectors are being explored in order to produce structures that overcome the limitations of conventional electrospun structures. The range of techniques developed based on solid mechanical collectors and the corresponding electrospun 3D structure obtained are discussed below.

A rotating mandrel collector has been utilised for producing tubular structures [45]. By optimising the mandrel's rotating speed, aligned fibres were produced which are beneficial for neural TE applications. 
While at lower speeds, the rotation is unable to align the fibres on its surface along the direction o frotation, at higher speeds the collection of the fibres itself is disrupted. At the optimised rotating speed, the deposition of the fibres is matched with the rotation of the surface and they are guided and aligned along the direction of rotation.

A dry ice filled rotating drum, when used a collector has resulted in a cottonwool like structure with elevated compressibility and mouldablity appropriate for bone filling applications. A highly permeable tissue graft has been fabricated using a perforated mandrel through which pressurized air can exit [46]. This graft displayed sufficient mechanical properties and superior cellular penetration than the scaffolds got with the help of a non-perforated mandrel. Fibre accumulation has been observed in the airgaps of two conducting structures. These include rings [43], poles [47] or blades [48]. While in a few cases bundles of aligned fibres $[44,45]$ were reported, others reported twisted mutifilament yarns [46] when one end of the fibre bundle is kept satationary and the other end is rotated. This technique has been referred to as airgap electrospinning. The intra-fibre distance also makes them suitable to function as nerve conduits. Modified collectors with a feature for coating rotating filaments with electrospun fibres, have also been developed [6] [47]. Such setups have been utislised for forming thick yarn like scaffolds that seemed to mimic the osteon structure in bones [6]. The presence of multiple conducting points positioned at the collector assembly has succeeded in producing fluffy structures. A hemispherical collector augmented with several conducting points placed inside it [48] and a collector with an arrangement of equidistant point electrodes at the outer edge of an open-ended frame made from flexible metallic rods [49] are examples of such collectors. In both cases the electrospun fibres were collected into a fluffy cotton ball like fibrous mass, between the conducting points. Also, in the latter scenario, when the collector was rotated and spinnerets of opposite polarities (positioned at $45^{\circ}$ ) were used, composite or core sheath yarns were electrospun.

\section{CONCLUSION}

The paper outlines the basics of bone biology before delving into the various reasons for bone loss. The role of TE in combating the global issue of bone loss is elaborated. The immense potential of electrospinning in fabricating self supporting 3D structures, for tissue regenerative applications, is yet to be explored in detail. Simple yet innovative methodologies reported in literature, are constantly expanding the horizon of electrospinning 3D structures. Additional work in this direction of novel collectors for electrospun fibres is bound to result in novel nano structures with applications in the medical and energy sectors..

\section{REFERENCES}

1. I. Binderman, Y. Avinoam, S. Yuval, B. Hila, C. Joseph and R. Philippe, "Tissue engineering of bone: critical evaluation of scaffold selection." In Bone Regeneration, edited by Haim Tal, 340. InTech. 2012.

2. U. Kneser, D. J. Schaefer, E. Polykandriotis and R. E. Horch, "Tissue engineering of bone: the reconstructive surgeon's point of view." Journal of Cellular and Molecular Medicine, vol. 10, no. 1, pp. 7-19. 2006
3. R. F. -Silva, G. R. Silva Sasso, E. S. -Cerri, M. J. Simões, and P. S. Cerri. "Biology of bone tissue: structure, function, and factors that influence bone cells." BioMed Research International, vol. 2015, pp. 1-17, 2015

4. P. Downey and I. S. Michael, "Bone Biology and the Clinical Implications for Osteoporosis.” Physical Therapy, vol. 86, no. 1, pp. 77-91, 2006.

5. Y. Hong, H. Fan, B. Li, B. Guo, M. Liu and X. Zhang, "Fabrication, biological effects, and medical applications of calcium phosphate nanoceramics." Materials Science and Engineering R: Reports vol. 70, no. 3, pp. 225-242, 2010.

6. T. Andric, A.C. Sampson, and J. W. Freeman, "Fabrication and characterization of electrospun osteon mimicking scaffolds for bone tissue engineering." Materials Science and Engineering: C, vol. 31, no. 1, pp. 2-8, 2011.

7. C. Sobacchi, S. Ansgar, F. P. Coxon, A. Villa and M. H. Helfrich, M. H. "Osteopetrosis: genetics, treatment and new insights into osteoclast function." Nature Reviews Endocrinology, vol. 9, no. 9, pp. 522-536, 2013.

8. T. C. A. Phan, J. Xu, and M. H. Zheng, "Interaction between osteoblast and osteoclast: impact in bone disease." Histology and Histopathology, vol. 19, no. 4, pp. 1325-1344, 2004.

9. P. Ginestra, E. Ceretti and A. Fiorentino, A. "Electrospinning of poly-caprolactone for scaffold manufacturing: experimental investigation on the process parameters influence." Procedia CIRP, vol. 49, pp. 8-13, 2016.

10. J. H. Jang, C. Oscar H. W. Kim, "Electrospun materials as potential platforms for bone tissue engineering." Advanced Drug Delivery Reviews, vol. 61, no. 12, pp. 1065-1083, 2009.

11. R. A. A. Muzzarelli, "Chitosan composites with inorganics, morphogenetic proteins and stem cells, for bone regeneration." Carbohydrate Polymers, vol. 83, no. 4, pp. 1433-1445, 2011.

12. T. Liu, W. Gang, Z. Yuanna, W. Daniel, W., Vincent, E. and Yuelian, L. "Cell-mediated bmp-2 release from a novel dual-drug delivery system promotes bone formation." Clinical Oral Implants Research, vol. 25, no. 12, pp. 1412-1421, 2014.

13. D. Arcos and M. Vallet-Regí, "Bioceramics for drug delivery." Acta Materialia vol. 61, no. 3, pp. 890-911, 2013.

14. B. Guillaume, "Filling bone defects with $\beta$-TCP in maxillofacial surgery: a review." Morphologie, vol. 101, no. 334, pp. 113-119, 2017.

15. M. Vallet-Reg1 and D. Arcos, Nanoceramics in Clinical Use. In RSC Nanoscience \& Nanotechnology. Cambridge: Royal Society of Chemistry, 2015.

16. W. I. Abdel-Fattah, F. M. Reicha and T. A. Elkhooly, "Nano-beta-tricalcium phosphates synthesis and biodegradation: 1. Effect of microwave and so42- ions on $\beta$-tcp synthesis and its characterization." Biomedical Materials, vol. 3, no. 3, pp. 034121, 2008

17. Starly, B., W. Lau, T. Bradbury, and W. Sun. "Internal architecture design and freeform fabrication of tissue replacement structures." Computer Aided Design, vol. 38, no. 2, pp. 115-124, 2006.

18. M. E. Kolewe, H. Park, C. Gray, X. Ye, R. Langer and L. E. Freed, “3D structural patterns in scalable, elastomeric scaffolds guide engineered tissue architecture." Advanced Materials, vol. 25, no. 32, pp. 4459-4465, 2013

19. M. Pilia, T. Guda, B. E. Pollot, , V. Aguero and M. R. Appleford, "Local microarchitecture affects mechanical properties of deposited extracellular matrix for osteonal regeneration." Materials Science and Engineering: C, vol. 35, no. 1, pp. 122-133, 2014.

20. J. Lee, M. J. Cuddihy and N. A. Kotov, "Three-dimensional cell culture matrices: state of the art." Tissue Engineering Part B: Reviews , vol. 14, no. 1, pp. 61-86, 2008.

21. K. M. Pawelec, A. Husmann, S. M. Best and R. E. Cameron, "Understanding anisotropy and architecture in ice-templated biopolymer scaffolds." Materials Science and Engineering: C, vol. 37, no. 1, pp. 141-147, 2014.

22. C. Liu, Z. Xia, and J. T. Czernuszka, "Design and development of three-dimensional scaffolds for tissue engineering." Chemical Engineering Research and Design, vol. 85, no. 7A, pp. 1051-1064, 2007.

23. K. Saha, J. F. Pollock, D. V. Schaffer and K. E. Healy, "Designing synthetic materials to control stem cell phenotype." Current Opinion in Chemical Biology, vol. 11, no. 4, pp. 381-387, 2007.

24. M. W. Tibbitt and K. S. Anseth, "Hydrogels as extracellular matrix mimics for $3 \mathrm{~d}$ cell culture." Biotechnology and Bioengineering, vol. 103, no. 4, pp. 655-663, 2009.

25. P. X. Ma "Scaffolds for tissue fabrication." Materials Today, vol. 7, no 5, pp. 30-40, 2004. 
26. N. Han, J. K. Johnson, A. B. Patrick, S. P. Kunal, J. L. John and J. O. Winter, "Cell attachment to hydrogel-electrospun fiber mat composite materials." Journal of Functional Biomaterials, vol. 3, no. 4, pp. 497-513, 2012.

27. S. H. Teng, W. Peng and H. E. Kim, "Blend fibers of chitosan-agarose by electrospinning." Materials Letters, vol. 63, no. 28, pp. 2510-2512, 2009.

28. Y. C. Toh, N. Susanne, M. K. Yuet, Z. Xin, Z. Yajuan, C. L. Pao, M. T. Chee, S. Wanxin and $\mathrm{H}$. Yu, "Cellular responses to a nanofibrous environment." Nano Today, vol. 1, no. 3, pp. 34-43, 2006.

29. S. D. McCullen, R. Sangeetha, L. I. Clarke, and R. E. Gorga, "Nanofibrous composites for tissue engineering applications." Wiley Interdisciplinary Reviews: Nanomedicine and Nanobiotechnology, vol. 1, no. 4, pp. 369-390, 2009.

30. N. M. Neves, Electrospinning for Advanced Biomedical Applications and Therapies. Smithers Rapra Technology, 2012.

31. M. M. Munir, B. S. Adi, I. Ferry and O. Kikuo "Scaling law on particle-to-fiber formation during electrospinning." Polymer, vol. 50, no. 20, pp. 4935-4943, 2009.

32. N. Bock, , T. R. Dargaville, and M. A. Woodruff, "Electrospraying of polymers with therapeutic molecules: state of the art." Progress in Polymer Science, vol. 37, no. 11, pp. 1510-1551, 2012.

33. D. Ahirwal, A. Hébraud, R. Kádár, M. Wilhelm and G. Schlatter, "From self assembly of electrospun nanofibers to 3D cm thick hierarchical foams." Soft Matter, vol. 9, no. 11, pp. 3164, 2013.

34. B. Sun, Y. Z. Long, H. D. Zhang, L. Meng-Meng, J. L. Duvail, X. Y. Jiang and H. L. Yin, "Advances in three-dimensional nanofibrous macrostructures via electrospinning." Progress in Polymer Science, vol. 39, no. 5, pp. 862-890, 2014.

35. M. Yousefzadeh, L. Masoud, A. T. Mohammad, W. E. Teo and S. Ramakrishna, "A note on the 3D structural design of electrospun nanofibers." Journal of Engineered Fibers and Fabrics, vol. 7, no. 2, pp. 17-23, 2012.

36. I. K. Shim, W. H. Suh, S. Y. Lee, S. H. Lee, S. J. Heo, M. C. Lee and S J. Lee, "Chitosan nano-/microfibrous double-layered membrane with rolled-up three130 dimensional structures for chondrocyte cultivation.' Journal of Biomedical Materials Research Part A, vol. 90A. no. 2, pp. 595-602, 2009.

37. Q. P. Pham, U. Sharma and A. G. Mikos, "Electrospun poly( $\varepsilon$-caprolactone) microfiber and multilayer nanofiber/microfiber scaffolds: characterization of scaffolds and measurement of cellular infiltration." Biomacromolecules, vol. 7, no. 10, pp. 2796-2805, 2006.

38. S. Soliman, P. Stefania, R. Antonio, F. Giancarlo, F. Roberta, P. Francesca, F. Ornella, "Multiscale three-dimensional scaffolds for soft tissue engineering via multimodal electrospinning." Acta Biomaterialia, vol. 6, no. 4, pp. 1227-1237, 2010.

39. T. G. Kim, H. J. Chung and T. G. Park, "Macroporous and nanofibrous hyaluronic acid/collagen hybrid scaffold fabricated by concurrent electrospinning and deposition/leaching of salt particles." Acta Biomaterialia, vol. 4, no. 6, pp. 1611-1619, 2008.

40. [40] M. Yousefzadeh, L. Masoud, A. T. Mohammad, W. E. Teo and S Ramakrishna, "A note on the 3D structural design of electrospun nanofibers." Journal of Engineered Fibers and Fabrics, vol. 7, no. 2, pp. 17-23, 2012.

41. B. Sun, L. Yun-Ze, F. Yu, L. Meng-Meng, Z. Hong-Di, L. Wen-Jing and X. Tian-Xiang, "Self-assembly of a three-dimensional fibrous polymer sponge by electrospinning." Nanoscale, vol. 4, no. 6, pp. 2134, 2012.

42. D. Ahirwal, A. Hébraud, R. Kádár, M. Wilhelm, and G. Schlatter, "From self assembly of electrospun nanofibers to 3D $\mathrm{cm}$ thick hierarchical foams." Soft Matter, vol. 9, no. 11, pp. 3164, 2013.

43. Y. S. Lee and T. L. Arinzeh, 2011. "Electrospun nanofibrous materials for neural tissue engineering." Polymers, vol. 3, no. 4, pp. 413-426.

44. O. D. Schneider, W. F. Tobias, L. Stefan, E. Martin, R. S. Patrick and J. S. Wendelin, "In vivo and in vitro evaluation of flexible, cottonwool-like nanocomposites as bone substitute material for complex defects." Acta Biomaterialia, vol. 5, no. 5, pp. 1775-1784, 2009.

45. M. J. McClure, S. W. Patricia, G. S. David, S. A. Sell, and G. L. Bowlin, "The use of air-flow impedance to control fiber deposition patterns during electrospinning." Biomaterials, vol. 33, no. 3, pp. 771-779, 2012

46. P. D. Dalton, K. Doris and M. Möller, "Electrospinning with dual collection rings." Polymer, vol. 46, no. 3, pp. 611-614, 2005.

47. A. Thorvaldsson, S. Hanna, G. Paul and W. Pernilla, "Electrospinning of highly porous scaffolds for cartilage regeneration." Biomacromolecules, vol. 9 , no. 3, pp. 1044-1049, 2008.

48. B. A. Blakeney, T. Ajay, M. A. Joel, A. Adinarayana, D. J. Lim, R. D. Derrick and W. J. Ho "Cell infiltration and growth in a low density, uncompressed three-dimensional electrospun nanofibrous scaffold." Biomaterials, vol. 32, no. 6, pp. 1583-1590, 2011.

49. J. Joseph, S. V. Nair and D. Menon, "Integrating substrateless electrospinning with textile technology for creating biodegradable three-dimensional structures." Nano Letters, vol. 15, no. 8, pp. 5420-5426, 2015

\section{AUTHORS PROFILE}

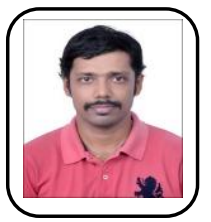

Dr. V. Yogeshwar Chakrapani is an associate professor at the department of Instrumentation and Control Engineering at Kalasalingam Academy of Research and Education, Krishnankoil, India. He has an interdisciplinary $\mathrm{PhD}$ in biomaterials for bone tissue engineering. He has published 6 peer reviewed journal papers and a book chapter. His research interest includes nanofibrous composite materials for medical applications.

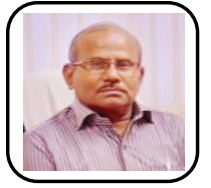

Dr. T. S. Sampath Kumar is a professor at the department of Metallurgical and Materials Engineering, IIT Madras. He heads the Medical Materials Laboratory. $\mathrm{He}$ has numerous publications to his credit in international journals. He has more than 20 years of experience in the teaching and research profession. His areas of interest include,

- Nano apatite based multi mode drug delivery \& imaging system

- Surface featuring of metallic implants for enhanced biofunctionalisation

- Electrospun and electrosprayed bioceramics and biocomposites

- Self-setting injectable bone and dental cements

- Antimicrobial materials, drug carriers and coatings. 